\title{
Influenza-attributable burden in United Kingdom primary care
}

\author{
D. M. FLEMING ${ }^{1}$, R. J. TAYLOR ${ }^{2}$, F. HAGUINET ${ }^{3}$, C. SCHUCK-PAIM ${ }^{2}$, \\ J. LOGIE ${ }^{4}$, D. J. WEBB ${ }^{4}$, R. L. LUSTIG ${ }^{2}$ AND G. MATIAS ${ }^{3}$ \\ 19 Dowles Close, Birmingham, UK (independent consultant) \\ ${ }^{2}$ Sage Analytica, Bethesda, MD, USA \\ ${ }^{3}$ GSK Vaccines, Wavre, Belgium \\ ${ }^{4}$ GSK R\&D, Uxbridge, Middlesex, UK
}

Received 19 December 2014; Final revision 1 April 2015; Accepted 6 May 2015; first published online 13 July 2015

\section{SUMMARY}

Influenza is rarely laboratory-confirmed and the outpatient influenza burden is rarely studied due to a lack of suitable data. We used the Clinical Practice Research Datalink (CPRD) and surveillance data from Public Health England in a linear regression model to assess the number of persons consulting UK general practitioners (GP episodes) for respiratory illness, otitis media and antibiotic prescriptions attributable to influenza during 14 seasons, 1995-2009. In CPRD we ascertained influenza vaccination status in each season and risk status (conditions associated with severe influenza outcomes). Seasonal mean estimates of influenza-attributable GP episodes in the UK were 857996 for respiratory disease including 68777 for otitis media, with wide inter-seasonal variability. In an average season, $2 \cdot 4 \% / 0 \cdot 5 \%$ of children aged $<5$ years and $1 \cdot 3 \% / 0 \cdot 1 \%$ of seniors aged $\geqslant 75$ years had a GP episode for respiratory illness attributed to influenza A/B. Two-thirds of influenza-attributable GP episodes were estimated to result in prescription of antibiotics. These estimates are substantially greater than those derived from clinically reported influenza-like illness in surveillance programmes. Because health service costs of influenza are largely borne in general practice, these are important findings for cost-benefit assessment of influenza vaccination programmes.

Key words: Epidemiology, GP surveillance systems, infectious disease, influenza, influenza vaccines.

\section{INTRODUCTION}

In most winters, influenza causes substantial morbidity and mortality [1, 2], with widely variable clinical manifestations ranging from subclinical infection to fatal illness [3]. Although many persons become infected, seroprevalence studies indicate that only a minority make use of health services [4]. Nevertheless, those that do create considerable pressure on both primary-

\footnotetext{
* Author for correspondence: D. M. Fleming, 9 Dowles Close, Birmingham, B29 4LE, UK.

(Email: dmfleming9dc@btinternet.com)
}

and secondary-care services $[5,6]$. The risk of serious illness involving hospital admission or death is thought to be higher in persons with chronic illness [7], but the total impact of influenza on health services comes at least as much from otherwise healthy persons [8]. In the UK this impact is concentrated in a comparatively short epidemic period of around 8 weeks [9], creating surge pressures on health services, commonly in midwinter, when the impact of other respiratory viral infections is also highest [10].

Policies to prevent and treat influenza need to accommodate the varying threat from different virus strain types, changes in severity of epidemics over time,

This is an Open Access article, distributed under the terms of the Creative Commons Attribution-NonCommercial-NoDerivatives licence (http://creativecommons. org/licenses/by-nc-nd/4.0/), which permits non-commercial re-use, distribution, and reproduction in any medium, provided the original work is unaltered and is properly cited. The written permission of Cambridge University Press must be obtained for commercial re-use or in order to create a derivative work. 
advances in vaccines, the ageing population, increasing population expectancies from healthcare and changes in healthcare delivery. These factors interact to influence the cost effectiveness of medical intervention, and create the need to update burden assessments on a continuing basis using measurements from several seasons.

Most estimates of the influenza burden employ modelling methods in which the identification of the influenza epidemic period is determined from available virology data and the impact measured in the commensurate changes in the chosen outcome [11-15]. In some modelling studies, influenza virus data have been used exclusively, but the confounding effect of other viruses has prompted the inclusion of data for other viruses, especially respiratory syncytial virus (RSV). In a few studies, meteorological data has been incorporated as well $[13,14]$. This study aims to provide a comprehensive analysis of the burden of persons consulting a general practitioner (GP) for a cause attributable to influenza over many seasons, to inform cost-benefit analyses of vaccination and treatment interventions. We used consultation data from the Clinical Practice Research Datalink (CPRD) and virology data from Public Health England (PHE) in a linear regression model to estimate influenza-attributable consultations and antibiotic prescriptions over 14 seasons, while controlling for RSV and other seasonal variables, and stratifying the analyses by age, comorbidity risk and influenza vaccination status.

\section{METHODS}

\section{Study design}

Viral surveillance time-series regression models were used to estimate the number of persons consulting a GP for influenza-attributable respiratory disease in England in each season (July in the index year through June the following year), from 1995 to 2009, stratifying by age, risk status and vaccination status (www. clinicaltrials.gov: NCT01520935). Seasons after July 2009 were not included as we were concerned to establish the normal or average seasonal burden of influenza, which was disturbed by the pandemic experience in summer 2009.

\section{Data sources}

At the midpoint of the study (January 2001) the monitored population was around 3.7 million. Patients included in the study were registered in approximately
550 general practices [16]. Individual patients could be followed for varying durations during this period. CPRD diagnoses and interventions are summarized and stored as READ codes.

Weekly influenza and RSV counts were obtained from PHE. Influenza reports are based on regular returns of virus swab-positive tests to PHE, mainly from community-based investigation of persons with influenza-like illness. RSV reports are mainly confirmed infections in young children admitted to hospital with respiratory infections. We used only counts of positive nose/throat swabs or nasopharyngeal aspirates. Viruses were classified as A or B without further classification.

All analyses of CPRD were restricted to English practices to match the coverage of virology data.

\section{Outcome definitions}

A GP episode was counted as the first in a series of consultations for a particular diagnosis/diagnostic group. A minimum of 28 days was required following any previous consultation for that same diagnosis/ diagnostic group before defining a new episode.

Diagnostic READ codes were combined into respiratory diagnoses consistent with recognized ICD10 groupings (Table 1) (see Supplementary material). We undertook a preliminary search of records to find codes that increased during influenza active periods. Our 'respiratory disease broadly defined' outcome included all disorders in the ICD-10 respiratory disease chapter, otitis media, as well as selected presenting symptom codes we found were used by some GPs in lieu of specifying a clinical diagnosis. A negative control outcome (urinary tract infection) with no seasonal pattern and association with influenza was included to control for the possibility of bias in model attribution over the entire study period.

Time-series of numbers of prescriptions of broad spectrum penicillin, tetracycline and macrolide antibiotics, which are widely prescribed for acute respiratory infections, were also examined as an outcome category.

\section{Vaccination and risk status}

The CPRD dataset contains unique patient identifiers, allowing patients to be followed longitudinally. Since almost all influenza vaccination during the study period was undertaken by GPs and recorded using specific codes, we were able to check the vaccination 
Table 1. Outcomes: general practitioner consultations (CPRD)*

\begin{tabular}{ll}
\hline \hline Outcome & ICD-10 codes \\
\hline $\begin{array}{l}\text { Respiratory diseases } \\
\text { Respiratory disease }\end{array}$ & J00-99, R05-06, B34, \\
$\begin{array}{l}\text { broadly defined: } \text { Resp } \\
\text { dis + symptoms + sepsis }\end{array}$ & A40-41, P36 \\
Respiratory disease & J00-99 \\
Acute upper respiratory & J00, J02-06 \\
disease & \\
Pneumonia and influenza & J09-18 \\
Bronchitis/bronchiolitis & J20-22, J40 \\
Influenza-like illness & J09-11, B34 \\
Non-respiratory diseases & \\
Otitis media & H65-66, H70 \\
Urinary tract infection & N39 \\
Drug prescriptions & \\
Antibiotics (broad & \\
spectrum penicillins, & \\
macrolides, & \\
tetracyclines) & \\
Risk factor definitions & Chronic heart disease; renal; \\
& diabetes (diagnosis/ \\
& monitoring); liver; stroke/ \\
TIA; MS-CNS; & COPD_no_asthma; asthma; \\
\hline \hline & immuno; \\
immuno_suppressant & treatment; anti-asthmatic \\
& \\
\hline
\end{tabular}

* Any Clinical Practice Research Datalink (CPRD) GP episode, including office visits, home visits, telephone consults and other types, for subjects registered with research quality data in CPRD. CPRD diagnostic data are coded using READ codes selected to correspond to ICD-10 definitions.

status for each person in each season. Vaccine coverage in the CPRD population was used to estimate the vaccination coverage in each age-risk stratum. We also externally validated the CPRD vaccination coverage estimates against national data on vaccination coverage [17]. We classified each patient as 'high' or 'low' risk by the prior occurrence of diagnoses that would prioritize the patient to receive influenza vaccination by reference to the accumulated historical records in the database (Table 1) [18].

\section{Denominators}

The CPRD covers a population which is representative of England in age and gender distribution and is thus a suitable population-denominated database for the assessment of vaccination status and national patterns of comorbidity in age- and gender-specific groups
[19]. We found excellent agreement between CPRD and independent data sources for the UK with respect to the population age structure, the prevalence of various risk factors, and influenza vaccination coverage; for example, CPRD data yielded an average vaccination rate of $71 \%$ for persons aged $\geqslant 65$ years for the years 2001-2008, in close agreement with PHE estimates [17]. The 2001 UK population (Office of National Statistics [20]) was thus used to weight the English CPRD population by age to reflect the UK profile.

\section{Statistical methods}

Statistical analyses were performed using SAS v. 9.2 (SAS Institute Inc., USA). Weekly time-series of the number of specimens positive for influenza A, influenza B and RSV were generated using PHE virology surveillance data. Weekly time-series for multiple influenza-related health outcomes (Table 1) were generated from the CPRD for age groups $<5$, $5-17,18-49,50-64$, and $\geqslant 65$ years (for selected analyses age groups $65-74$ and $\geqslant 75$ were examined separately) and low-/high-risk groups. A multiple linear regression model was applied to each age group to associate GP episodes to influenza A or B, while controlling for RSV and unspecified seasonal factors (Fig. 1). Major changes in virus detection methodology were undertaken by PHE in 2001; thus, to avoid bias the model separated the periods before and after this date:

$$
\begin{aligned}
Y= & \beta_{0}+\beta_{s 1} t+\beta_{s 2} t^{2}+\beta_{s 3} t^{3}+\beta_{s 4} \sin (2 \pi t / 52 \cdot 18) \\
& +\beta_{s 5} \cos (2 \pi t / 52 \cdot 18) \\
& +\beta_{p 1 a} \text { InfluenzaA(pre-July 2001) } \\
& +\beta_{p 1 b} \text { InfluenzaA (post-July 2001) } \\
& +\beta_{p 2 a} \text { InfluenzaB(pre-July 2001) } \\
& +\beta_{p 2 b} \text { InfluenzaB(post-July 2001) } \\
& +\beta_{p 3 a} \text { RSV(pre-July 2001) } \\
& +\beta_{p 3 b} \text { RSV(post-July 2001) }
\end{aligned}
$$

where, $Y$ is the incidence (rate) of outcome definition for each time period $t$ (weeks), RSV and influenza are the proportion of laboratory isolates during $t$, sin and $\cos$ are harmonic functions of $t$, and the remaining terms track other types of secular trends in the data. The time period $t$ used in the model is a running index of weeks starting 1 October 1997 and ending 31 March 2009. 


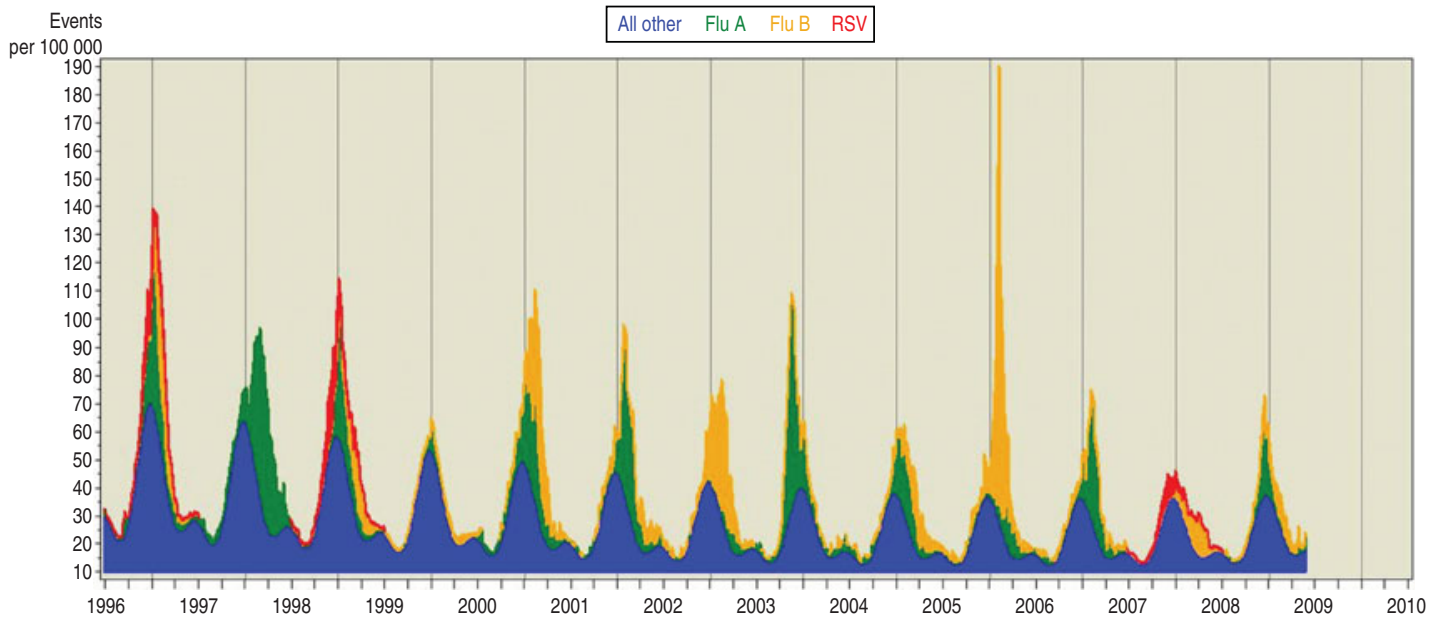

Fig. 1. Attribution modelling showing excess GP episodes attributable to influenza A and B and respiratory syncytial virus (RSV) in children aged 5-17 years. The pattern was observed to be the same for other age groups. 'All other' refers to GP episodes not attributable to influenza or RSV. The denominator is the UK population.

$\beta_{p 1 a}$ Influenza $\mathrm{A}\left(\right.$ pre-July 2001) $+\beta_{p 1 b}$ Influenza $\mathrm{A}$ (post-July 2001) are influenza terms pre- and post-July, 2001. The RSV terms control for RSV epidemics. Influenza A, Influenza B and RSV are observed counts of positive tests from the PHE LabBase dataset.

Outcome and pathogen series were tri-meansmoothed, i.e. the smoothed value of $X$ at week $t$ is $\left(X_{t-1}+X_{t}+X_{t+1}\right) / 3$. The week numbers $(t)$ were calculated as ISO $8601 \mathrm{~V}$-weeks. The weekly attribution to each virus was computed as the product of the observed value of the explanatory variable (i.e. number of positive virology samples) and the corresponding regression coefficient $\left(\beta_{p 1 a}-\beta_{p 2 b}\right)$, and the weekly estimates summed to produce the seasonal estimates. The variability of seasonal estimates was assessed using standard deviations but limited to all-age estimates. This standard deviation represents the variability of the attributable burden between seasons and not the uncertainty of the individual seasonal estimates. Results were expressed as numbers of persons consulting a GP (GP episodes) and as the mean seasonal (from September to mid-May) rates of persons consulting a GP per 100000 population.

Outside pandemics, influenza is exclusively a winter illness and thus summertime data were excluded from the analysis. Previous evidence suggests that winter increases in the incidence of RSV-associated outcomes (e.g. acute bronchitis) in adults follow those in children by 2-3 weeks [1]. Therefore, for age groups aged $\geqslant 18$ years, we lagged the RSV time-series by 2 weeks and achieved an improved model fit; we did not lag the influenza time-series as doing so did not substantially alter model fit or influenza burden attributions. The time-series of prescriptions of antibiotics was modelled in the same way as the health outcome time-series to estimate antibiotic prescribing attributable to influenza. The model did not attribute any positive influenza burden to the control outcome (urinary tract infection).

The protocol was approved by the Independent Scientific Advisory Committee of the Clinical Practice Research Datalink (CPRD, formerly the General Practice Research Database). Informed consent was not required.

\section{RESULTS}

\section{Time trends}

The onset of the influenza epidemic period usually followed RSV, and influenza A usually preceded influenza B (illustrated for the 5-17 years age group in Fig. 1).

\section{Model fit}

The goodness-of-fit was assessed using adjusted $R^{2}$. The model fit in most strata was very good, with adjusted $R^{2}$ between 0.75 and 0.86 for the respiratory broad outcome (Supplementary Table S1). Moreover, we found a substantial 'lift' in the adjusted $R^{2}$ value when introducing the virology terms into the base secular model. We did not adjust the model form (by including or excluding terms) individually for 
Table 2. Persons consulting a general practitioner in a mean season (number and ratel100 000 population) attributable to influenza in the UK, by age (1995-2009)

\begin{tabular}{|c|c|c|c|c|c|}
\hline \multirow[b]{2}{*}{ Outcome } & \multirow[b]{2}{*}{ Age, yr } & \multicolumn{2}{|l|}{ Influenza A } & \multicolumn{2}{|l|}{ Influenza B } \\
\hline & & $n$ (S.D.) & Rate (S.D.) & $n$ (S.D.) & Rate (S.D.) \\
\hline \multirow{6}{*}{$\begin{array}{l}\text { Respiratory disease } \\
\text { broadly defined }\end{array}$} & $0-4$ & 84806 & 2436 & 18781 & 539 \\
\hline & $5-17$ & 120151 & 1217 & 98380 & 996 \\
\hline & $18-49$ & 234476 & 899 & 75160 & 288 \\
\hline & $50-64$ & 110768 & 1076 & 18386 & 179 \\
\hline & $\geqslant 65$ & 102513 & 1140 & 6810 & 76 \\
\hline & All ages & $649219(366681)$ & $1105(624)$ & 208777 (218 110) & $355(371)$ \\
\hline \multirow{6}{*}{$\begin{array}{l}\text { Bronchitis and } \\
\text { bronchiolitis }\end{array}$} & $0-4$ & 10027 & 288 & 0 & 0 \\
\hline & $5-17$ & 17078 & 173 & 10058 & 102 \\
\hline & $18-49$ & 64179 & 246 & 18774 & 72 \\
\hline & $50-64$ & 50640 & 492 & 6603 & 64 \\
\hline & $\geqslant 65$ & 63621 & 707 & 3735 & 42 \\
\hline & All ages & $210607(137270)$ & $359(234)$ & $38120(46417)$ & $65(79)$ \\
\hline \multirow[t]{6}{*}{ Influenza-like illness } & $0-4$ & 21955 & 631 & 4260 & 122 \\
\hline & $5-17$ & 33153 & 336 & 19103 & 193 \\
\hline & $18-49$ & 91202 & 350 & 25954 & 99 \\
\hline & $50-64$ & 38045 & 369 & 6100 & 59 \\
\hline & $\geqslant 65$ & 25589 & 284 & 2110 & 23 \\
\hline & All ages & 209154 (146 553) & $356(250)$ & 55969 (61 902) & $95(105)$ \\
\hline \multirow{6}{*}{$\begin{array}{l}\text { Pneumonia and } \\
\text { influenza }\end{array}$} & $0-4$ & 8923 & 256 & 1247 & 36 \\
\hline & $5-17$ & 19991 & 202 & 8978 & 91 \\
\hline & $18-49$ & 73273 & 281 & 20181 & 77 \\
\hline & $50-64$ & 32394 & 315 & 4521 & 44 \\
\hline & $\geqslant 65$ & 25722 & 286 & 2158 & 24 \\
\hline & All ages & 161228 (123 129) & $274(210)$ & 36541 (43 993) & $62(75)$ \\
\hline \multirow[t]{6}{*}{ Otitis media } & $0-4$ & 18202 & 523 & 6589 & 189 \\
\hline & $5-17$ & 19093 & 193 & 15041 & 152 \\
\hline & $18-49$ & 8816 & 34 & 2924 & 11 \\
\hline & $50-64$ & 878 & 9 & 1052 & 10 \\
\hline & $\geqslant 65$ & 158 & 2 & 315 & 3 \\
\hline & All ages & $44534(25065)$ & $76(43)$ & 24243 (28 284) & $41(48)$ \\
\hline \multirow{6}{*}{$\begin{array}{l}\text { Antibiotic } \\
\text { prescriptions }\end{array}$} & $0-4$ & 49759 & 1429 & 12130 & 348 \\
\hline & $5-17$ & 69008 & 699 & 59427 & 602 \\
\hline & $18-49$ & 159650 & 612 & 54858 & 210 \\
\hline & $50-64$ & 93806 & 911 & 15286 & 148 \\
\hline & $\geqslant 65$ & 88172 & 980 & 5547 & 62 \\
\hline & All ages & $461839(262785)$ & $786(447)$ & 142203 (162 168) & $242(276)$ \\
\hline
\end{tabular}

$n$, Number of episodes; S.D., standard deviation.

each outcome, age, and risk stratum, but applied the given form across all strata.

\section{Overall disease burden}

In a mean season there were an estimated $857996 \mathrm{GP}$ episodes in persons of all ages $(1 \cdot 5 \%$ of the population) for influenza-attributable respiratory disease (broadly defined), of which around 75\% (649 219) were for influenza A (Table 2). There was wide interseasonal variability in influenza $\mathrm{A}$ - and $\mathrm{B}$-attributable GP episodes, compared to a more stable consulting rate for RSV (Fig. 2). In the seasons studied the number of GP episodes for an influenza-attributable respiratory disease ranged between 174305 (20022003) and 1340937 (1995-1996) for influenza A, and between 2075 (1997-1998) and 646457 (19961997) for influenza B. The highest rates of GP episodes for influenza A-attributable respiratory disease were observed in children aged $<5$ years (Table 2). The highest seasonal incidence rates for influenza B were observed in those aged 5-17 years.

The rate of GP episodes for influenza A-attributable respiratory disease tended to increase after age 50 years, 


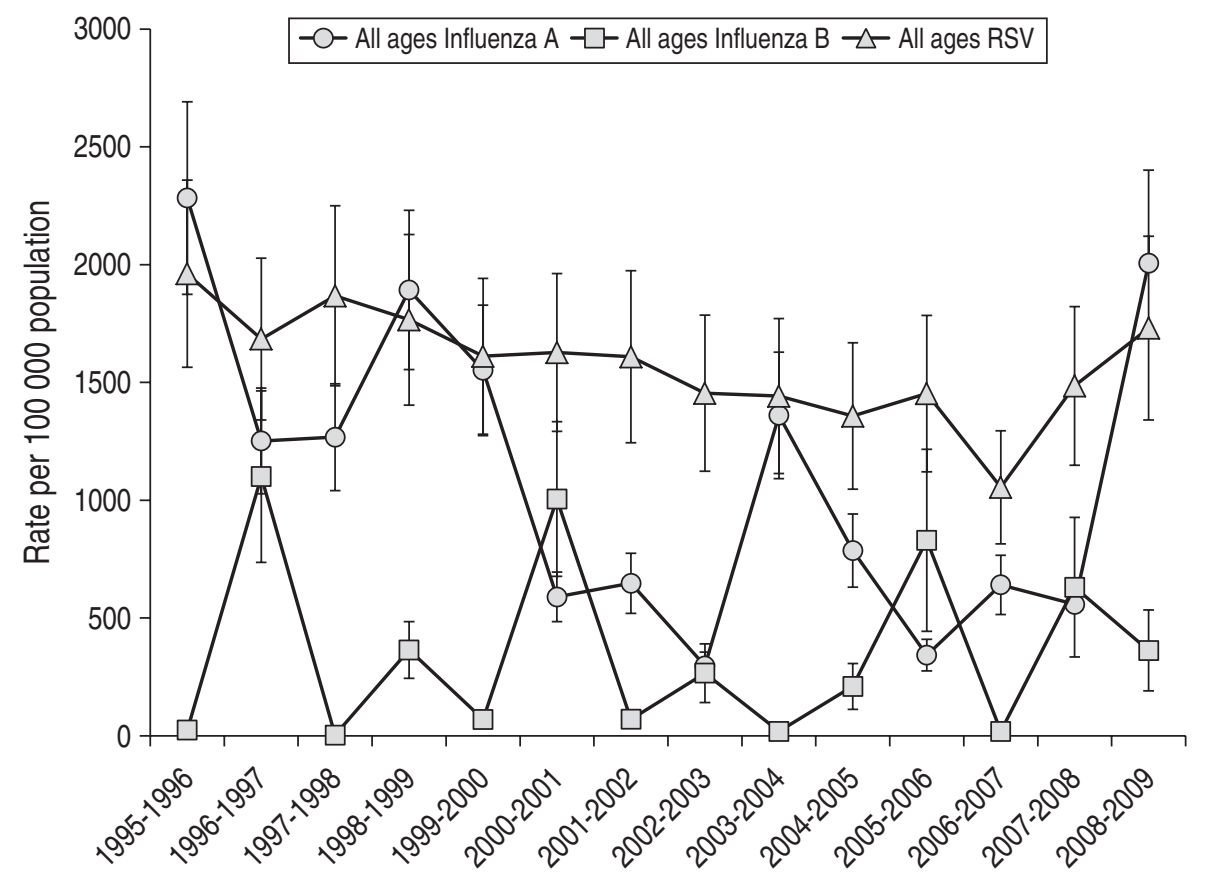

Fig. 2. Seasonal rates (with $95 \%$ confidence intervals) of GP episodes for respiratory disease (broadly defined) attributable to influenza A, influenza B and respiratory syncytial virus (RSV).

whereas GP episodes for influenza B-attributable respiratory disease decreased with age (Table 2). In the $\geqslant 75$ years age group, the rate of GP episodes for respiratory disease was $1255 / 100000$ for influenza A and $93 / 100000$ for influenza B.

In a mean season, the rate of GP episodes for influenza A-attributable bronchitis was higher in adults than in children, whereas the rate of GP episodes for influenza-like illness was higher in children than adults (Table 2). The rates of influenza A-attributable GP episodes for 'pneumonia and influenza' were higher in adults than in children. In those aged $\geqslant 75$ years, the mean seasonal rate of GP episodes for 'pneumonia and influenza' was 309/100 000 for influenza A, and 24/100 000 for influenza B.

\section{Otitis media and antibiotic use}

Influenza A and B contributed to 68777 GP episodes for otitis media per season, with the highest number in children (Table 2). Around one third of the GP episodes for otitis media were attributed to influenza B.

There were 604042 prescriptions for antibiotics attributed to influenza-associated respiratory disease (as measured in the broad respiratory outcome) per season across all age groups (Table 2).

\section{Comorbid risk status and vaccination}

Individuals with comorbid conditions that put them at high risk of severe influenza infection were more likely to consult a GP for an influenza-attributable respiratory disease than individuals at low risk (Table 3). The exceptions were children aged $<5$ years for influenza $\mathrm{A}$, and adults aged 50-64 years for influenza $B$. The average seasonal rates of influenza A-attributable GP episodes in the unvaccinated high comorbid risk group were 1.79-, 1.56- and 1.83-fold higher than in the low comorbid risk group for older adults aged 50 $64,65-74$ and $\geqslant 75$ years, respectively. Trends for influenza B were similar for the $65-74$ and $\geqslant 75$ years age groups (1.74- and 2.97-fold higher rates in high comorbid risk group, respectively).

Higher rates of GP episodes were observed in all age groups when comorbid risk factors were present than when they were absent for the other outcomes studied.

Between 2000 and 2008 influenza vaccine coverage in the CPRD population aged $\geqslant 65$ years ranged between $64 \%$ and $76 \%$, which was similar to coverage estimated by PHE [17]. In each season studied the rate of influenza A-attributable GP episodes in the high comorbid risk group aged 18-49 years was 0.6to $0 \cdot 89$-fold lower in vaccinated compared to unvaccinated individuals. The rate ratio of influenza 
Table 3. Persons consulting a general practitioner in a mean season (ratel100 000 population) for outcomes attributed to influenza $A$ and $B$ by comorbid risk status

\begin{tabular}{|c|c|c|c|c|c|}
\hline \multirow[b]{2}{*}{ Outcome } & \multirow[b]{2}{*}{ Age, yr } & \multicolumn{2}{|l|}{ Influenza A } & \multicolumn{2}{|l|}{ Influenza B } \\
\hline & & Low risk (s.D.) & High risk (s.D.) & Low risk (s.D.) & High risk (s.D.) \\
\hline \multirow[t]{6}{*}{ Respiratory broadly defined } & $0-4$ & 2482 & 1713 & 528 & 745 \\
\hline & $5-17$ & 1166 & 1562 & 953 & 1315 \\
\hline & $18-49$ & 831 & 1454 & 286 & 309 \\
\hline & $50-64$ & 948 & 1534 & 192 & 141 \\
\hline & $\geqslant 65$ & 889 & 1439 & 67 & 98 \\
\hline & All ages & 1024 (569) & $1464(920)$ & $374(390)$ & 289 (299) \\
\hline \multirow[t]{6}{*}{ Bronchitis and bronchiolitis } & $0-4$ & 279 & 417 & 0 & 95 \\
\hline & $5-17$ & 148 & 335 & 91 & 181 \\
\hline & $18-49$ & 212 & 523 & 69 & 98 \\
\hline & $50-64$ & 411 & 778 & 65 & 65 \\
\hline & $\geqslant 65$ & 516 & 934 & 31 & 60 \\
\hline & All ages & 275 (179) & 718 (496) & $62(74)$ & $82(103)$ \\
\hline \multirow[t]{6}{*}{ Influenza-like illness } & $0-4$ & 615 & 828 & 121 & 149 \\
\hline & $5-17$ & 316 & 465 & 186 & 252 \\
\hline & $18-49$ & 330 & 515 & 97 & 121 \\
\hline & $50-64$ & 339 & 482 & 60 & 55 \\
\hline & $\geqslant 65$ & 242 & 337 & 21 & 26 \\
\hline & All ages & $339(231)$ & $435(341)$ & 99 (109) & $81(91)$ \\
\hline \multirow[t]{6}{*}{ Pneumonia and influenza } & $0-4$ & 248 & 362 & 35 & 44 \\
\hline & $5-17$ & 189 & 295 & 88 & 110 \\
\hline & $18-49$ & 264 & 425 & 75 & 97 \\
\hline & $50-64$ & 285 & 425 & 45 & 41 \\
\hline & $\geqslant 65$ & 226 & 362 & 18 & 32 \\
\hline & All ages & 251 (187) & 383 (316) & $63(76)$ & $58(72)$ \\
\hline
\end{tabular}

S.D., Standard deviation.

A-attributable GP episodes in the high comorbid risk group in vaccinated over unvaccinated individuals was between 0.74 and 0.76 in those aged $50-64$ years, and $0 \cdot 81-0 \cdot 85$ in those aged $\geqslant 65$ years.

Similarly, in these age groups the rate of GP episodes was lower in vaccinated vs. unvaccinated persons at low risk: the rate ratio of influenza-A attributable GP episodes in the low comorbid risk group in vaccinated over unvaccinated was $0 \cdot 78-1$ in those aged 18-49 years, $0.79-0.92$ in those aged 50 64 years, and $0 \cdot 76-0 \cdot 98$ in those aged $\geqslant 65$ years.

\section{Clinical syndromes}

A wide spectrum of diseases that resulted in a GP episode was attributable to influenza A and B (Fig. 3). The relative proportions attributable to influenza $A$ and B varied with the GP-reported diagnoses, highlighting the importance of influenza $\mathrm{B}$ in the 5-17 years age group; especially so since influenza B seasons are less frequent than influenza A.

\section{DISCUSSION}

We used the CPRD in the UK, a promising but as yet underutilized nationally representative database for influenza burden assessment, to estimate the agespecific influenza burden in primary care [16]. We employed traditional methods used for hospitalization and mortality studies, and applied them to time-series of GP episodes for a diverse range of outcomes forming part of the burden of influenza, such as comorbidity risk status and antibiotic prescriptions, after taking account of the potentially confounding effects of RSV. In a mean season, around $1.5 \%$ of the UK population were estimated to present to GPs with diverse respiratory syndromes attributable to influenza A or B. The estimated mean seasonal burden of influenza A was much larger than influenza $B$ in all age groups and for most respiratory outcomes. Approximately 0.7\% of children aged $<5$ years were estimated to consult a GP for influenza-related otitis media in a mean season, and nearly $2 \%$ of all children received antibiotic prescriptions which were attributable to influenza. 

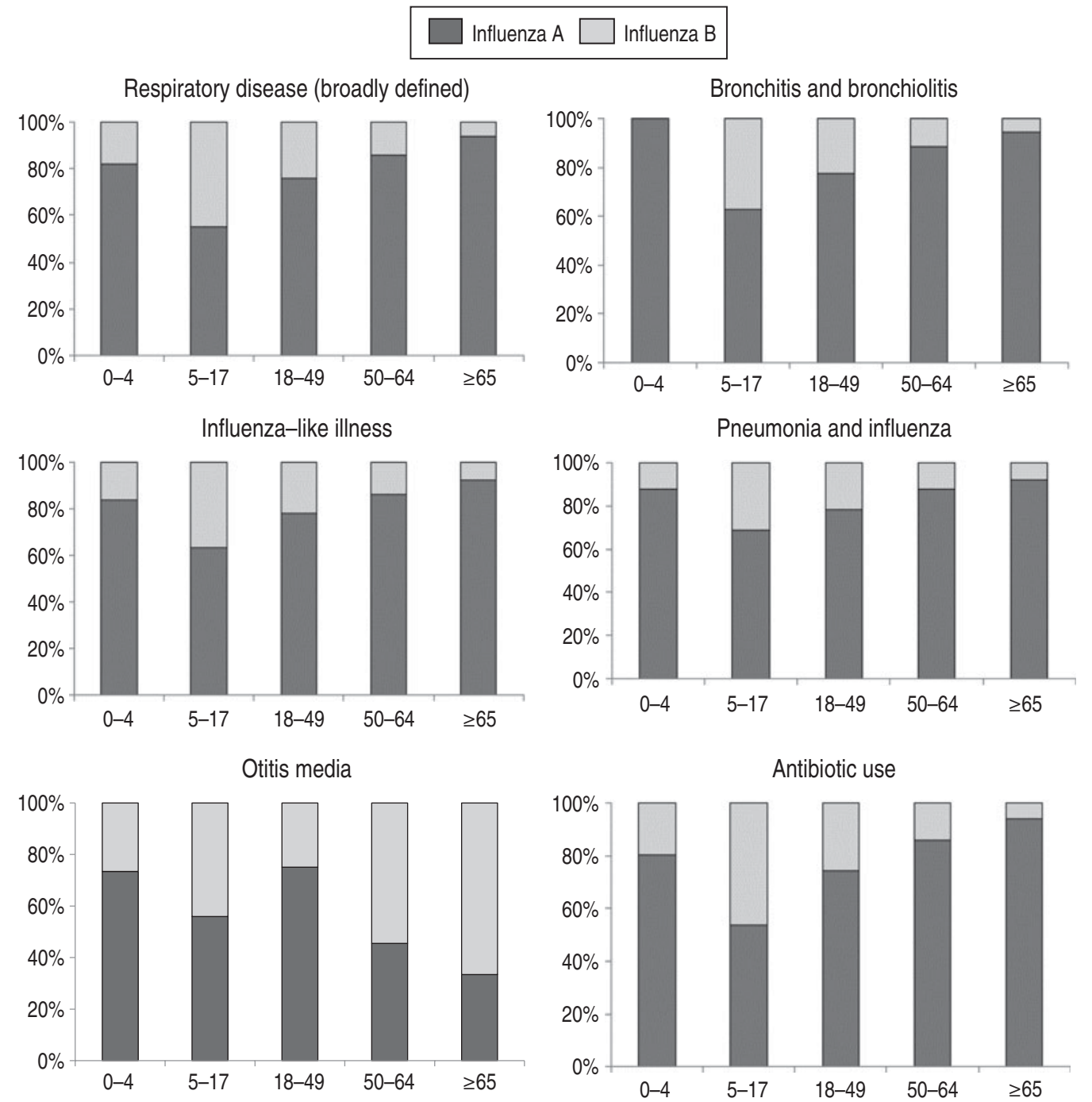

Fig. 3. The percentage of the estimated burdens for several outcomes attributed to influenza A and influenza B by five age groups (mean across seasons). The total number of GP episodes for each outcome was: respiratory disease (broadly defined) $(n=857996)$, bronchitis and bronchiolitis $(n=248727)$, influenza-like illness $(n=265123)$, pneumonia and influenza $(n=197769)$, otitis media $(n=68777)$, antibiotic use $(n=604042)$.

Compared to the relatively stable disease burden attributable to RSV in each season, the influenza burden was characterized by marked inter-seasonal variability according to epidemic activity.

The single previous UK study by Pitman et al. that used the CPRD data source to study GP office visits examined only a single season (2002-2003) [13]. In that study, Pitman et al. estimated approximately one million influenza-attributable consultations. This is higher than our estimate for that season of 330 763 GP episodes, but closer to our mean seasonal estimate encompassing 14 seasons. As well as the inherent instability of estimates produced using a single season, the model used by Pitman et al. [13] reported two alternative estimates obtained by models with and without a bacterial pathogen series included in the explanatory variables. The inclusion in the model of Pitman et al. of the bacterial series, characterized by a dominant sinusoidal pattern, likely affected the influenza burden estimate similarly to our use of cyclic components.

Estimates of the number of persons infected with influenza in an average season vary widely, but when measured as persons consulting with clinical illness they are mostly less than $5 \%$ of the population, depending on the diagnostic outcomes measured $[8,9,21,22]$. Our findings are consistent with a simpler analysis conducted in a database of the Royal 
College of General Practitioners, which estimated an influenza burden affecting $2 \cdot 1 \%$ of the population of England and Wales in seasons 1989-1999 [5].

We observed that in persons with comorbid conditions that placed them in a high risk group and in all persons aged $<65$ years, there were fewer GP episodes for influenza-attributable respiratory disease in vaccinated than non-vaccinated persons. This finding is difficult to interpret because our study was not designed to measure vaccine effectiveness, which would require a different study design. Such studies would require an analysis of the propensity to consult, which differs between vaccinated and non-vaccinated persons, and between those with high and those with low comorbidity risk.

Population-based studies of the influenza burden relying on individual laboratory-confirmed cases alone are not practical, due to the lack of standard testing, the expense of large-scale testing using sensitive PCR-based assays, and the fact that infections are often resolved by the time patients seek medical care (if they seek such care at all). Instead, indirect modelling approaches have been used for many years to estimate the burden of influenza [12, 23]. We used a previously described linear regression model [24], which adopted a recent refinement of regression techniques guided by virus surveillance data as described in studies by Pitman et al. [13] and Zhou et al. [25]. Other models used to estimate the burden of respiratory infections, such as Poisson regression with a natural logarithm for the link function $[11,26]$, imply multiplicative effects of respiratory viruses (i.e. the effect of multiple simultaneously circulating respiratory viruses is different from the sum of their individual effects). This is likely an unrealistic assumption for influenza strains [27, 28]. The linear regression model has the advantage over other methods of being an additive model, which corresponds to the view that the total burden in the population is due to the sum of outcomes due to different causes, including viral infections [27, 29].

The strengths of our model include the use of large nationally representative databases, which limited the potential for sampling error; inclusion of data over a long period that covered 14 seasons; and the use of control outcomes with no inherent seasonality and no association to influenza. By defining a new outcome category ('respiratory disease broadly defined'), we aimed to improve the sensitivity in capturing the full burden of influenza-attributable disease, while retaining sufficient specificity; for example, the additional codes in the 'respiratory broad' outcome increased the average seasonal all-age estimate by $14 \%$ over the stricter 'respiratory' outcome, but left the confidence intervals and the model fit virtually unchanged (data not shown). Age stratification allowed detailed estimation of the agespecific burden, controlled for the observed age effect on consultation rates and overcame possible confounding due to higher frequency of viral testing in some age groups. Finally, weekly occurrences of outcomes were assumed to be determined by the circulation of influenza, RSV, and other causes which follow a seasonality that was estimated using a combination of sine and cosine terms. The inclusion of both cyclical terms (sine and cosine functions) controlled for confounders for which data are not available. Tri-mean smoothing of pathogens and outcomes series mitigated short-term effects such as national holidays and extreme weather conditions on viral testing and GP consultations.

A potential limitation of the study is the exclusive analysis of influenza and RSV virus time-series with no consideration of other respiratory pathogens; if such pathogens consistently circulate at the same time as influenza, the influenza burden could be overestimated. We did not generate time-series for influenza subtypes as we did not have access to subtyped data. We did not evaluate the effect of pneumococcal vaccination in children or older age groups [30], or consider changes in herd immunity during the period of study, nor did we consider the effects of obesity [31, 32]. The study is also dependent on the quality and consistency of routine recording in the practice network. The burden of illness as encountered in primary care, which is the primary objective of this study, includes all consulting persons regardless of their propensity to consult. We did not study the indirect burden of illness in individuals with influenza who did not consult. While our study provides up-to-date and detailed information on health service utilization, the study does not provide information for evaluation of indirect medical costs associated with influenza. None of the potential limitations of the study are likely to have influenced the model estimates in any major way.

In addition to influenza-attributable respiratory disease, we estimated the burden of influenza-attributable otitis media, which has often been regarded as a common complication of influenza [33]. Our study found almost as many children (aged $<5$ years) were diagnosed at presentation with influenza-attributable otitis media as with influenza-like illness, suggesting that otitis 
media is part of the primary symptomatology of influenza. The diverse range of presenting illnesses identified as attributable to influenza in our study questions the relevance of diagnostic criteria by which influenza is defined clinically. Like other studies that have tried unsuccessfully to correlate specific symptoms with a diagnosis of influenza [34, 35], our study suggests that collections of symptoms with/without fever are largely inadequate for measuring the burden of illness.

Finally, the model estimated that more than 600000 antibiotic courses were prescribed for influenza-associated respiratory disease per season. The potential misuse of antibiotics is a well-recognized cause for concern, particularly in relation to common respiratory infections [36]. A simple test that is able to discriminate between bacterial and viral infection would be a great step forward, as would timely local influenza surveillance data that would allow physicians to assess the probability that an acute respiratory infection is caused by influenza.

Health economic models can inform policy choices, but they depend on accurate and age-specific parameter estimates of the disease burden. Furthermore, it is critical to use estimates based on recent assessments of the burden: earlier publications could overestimate the current burden. Because the effectiveness of influenza vaccination in preventing otitis media appears similar to that of pneumococcal conjugate vaccines [37], it is important that estimates of the otitis media burden and the potentially preventable fraction be included in cost-benefit estimates of influenza interventions in this age group. Our study provides a source for understanding the national influenza burden and undertaking cost evaluations.

\section{SUPPLEMENTARY MATERIAL}

For supplementary material accompanying this paper visit http://dx.doi.org/10.1017/S0950268815001119.

\section{ACKNOWLEDGEMENTS}

The authors thank the Sage Analytica team, in particular Lone Simonsen and Lewis Kim, for their contribution to the study. GlaxoSmithKline Biologicals SA was the funding source and was involved in all stages of the study conduct and analysis. GlaxoSmithKline Biologicals SA also took in charge all costs associated with the development and publication of the present manuscript. The authors are grateful to all teams of GSK Vaccines for their contribution to this study, especially Robert Gardner (scientific writer), Archana Jastorff (scientific writer; XPE Pharma and Science, on behalf of GSK Vaccines), Els Tassenoy (global study manager; Harrison Clinical Research, on behalf of GSK Vaccines), and Dominique Rosillon (Biometrics). Finally, the authors thank Joanne Wolter (independent medical writer, on behalf of GSK Vaccines) for providing writing services and Bruno Dumont (Business and Decision Life Sciences, on behalf of GSK Vaccines) for editorial assistance and manuscript coordination. Virology data were sourced from Public Health England (formerly Health Protection Agency). The opinions expressed in this report are solely those of the authors. [www.clinicaltrials.gov identifier: NCT01520935]

\section{DECLARATION OF INTEREST}

Gonçalo Matias, Dave J. Webb, John Logie, and François Haguinet are employed by the GSK Group of Companies. Dave J. Webb and John Logie report ownership of stock options and/or restricted shares. Robert J. Taylor, Roger L. Lustig, and Cynthia Schuck-Paim report having received consulting fees from Sage Analytica LLC to perform this study, paid for by the GSK Group of Companies. Douglas M. Fleming reports personal fees from Sage Analytica, personal fees and non-financial support from the GSK Group of Companies during the conduct of the study.

\section{REFERENCES}

1. Fleming DM, Elliot AJ. Lessons from 40 years' surveillance of influenza in England and Wales. Epidemiology and Infection 2008; 136: 866-875.

2. Fleming DM. The contribution of influenza to combined acute respiratory infections, hospital admissions, and deaths in winter. Communicable Disease and Public Health 2000; 3: 32-38.

3. Nicholson KG. Chapter 19. In: Nicholson KG, Webster RG, Hay AJ, eds. Textbook of Influenza. Oxford: Blackwell Sciences, 1998, pp. 221-223.

4. Miller E, et al. Incidence of 2009 pandemic influenza A H1N1 infection in England: a cross-sectional serological study. Lancet 2010; 375: 1100-1108.

5. Fleming DM, Zambon M, Bartelds AI. Population estimates of persons presenting to general practitioners with influenza-like illness, 1987-96: a study of the demography of influenza-like illness in sentinel practice networks in England and Wales, and in The Netherlands. Epidemiology and Infection 2000; 124: 245-253.

6. Fleming D, Harcourt S, Smith G. Influenza and adult hospital admissions for respiratory conditions in 
England 1989-2001. Communicable Disease and Public Health 2003; 6: 231-237.

7. Glezen WP, et al. Impact of respiratory virus infections on persons with chronic underlying conditions. Journal of the American Medical Association 2000; 283: 499-505.

8. Molinari N-AM, et al. The annual impact of seasonal influenza in the US: measuring disease burden and costs. Vaccine 2007; 25: 5086-5096.

9. Fleming DM, et al. The duration and magnitude of influenza epidemics: a study of surveillance data from sentinel general practices in England, Wales and the Netherlands. European Journal of Epidemiology 1999; 15: 467-473.

10. Elliot AJ, Cross KW, Fleming DM. Acute respiratory infections and winter pressures on hospital admissions in England and Wales 1990-2005. Journal of Public Health (Oxford) 2008; 30: 91-98.

11. Thompson WW, et al. Mortality associated with influenza and respiratory syncytial virus in the United States. Journal of the American Medical Association 2003; 289: 179-186.

12. Thompson WW, et al. Influenza-associated hospitalizations in the United States. Journal of the American Medical Association 2004; 292: 1333-1340.

13. Pitman RJ, et al. Assessing the burden of influenza and other respiratory infections in England and Wales. Journal of Infection 2007; 54: 530-538.

14. Hardelid P, Pebody R, Andrews N. Mortality caused by influenza and respiratory syncytial virus by age group in England and Wales 1999-2010. Influenza and Other Respiratory Viruses 2013; 7: 35-45.

15. Zambon MC, et al. Contribution of influenza and respiratory syncytial virus to community cases of influenza-like illness: an observational study. Lancet 2001; 358: 1410-1416.

16. Williams T et al. Recent advances in the utility and use of the General Practice Research Database as an example of a UK Primary Care Data resource. Therapeutic Advances in Drug Safety 2013; 3: 89-99.

17. Pebody RG, et al. Influenza vaccination coverage in England, 2000-2008. Eurosurveillance 2008; 13: 19074.

18. The Flu Immunisation Programme 2012/13Publications - GOV.UK (https://www.gov.uk/government /publications/the-flu-immunisation-programme-2012-13). Accessed 31 October 2013.

19. Walley T, Mantgani A. The UK General Practice Research Database. Lancet 1997; 350: 1097-1099.

20. Office of National Statistics. Taxonomy, 2010 (http:// www.ons.gov.uk/ons/taxonomy/index.html?nscl=Population+Estimates). Accessed 17 December 2013.

21. Sullivan KM, Monto AS, Longini IM. Jr Estimates of the US health impact of influenza. American Journal of Public Health 1993; 83: 1712-1716.

22. Schanzer DL, Schwartz B. Impact of seasonal and pandemic influenza on emergency department visits, 2003-
2010, Ontario, Canada. Academic Emergency Medicine 2013; 20: 388-397.

23. Serfling RE. Methods for current statistical analysis of excess pneumonia-influenza deaths. Public Health Report 1963; 78: 494-506.

24. Matias G, et al. Estimates of mortality attributable to influenza and RSV in the United States during 19972009 by influenza type or subtype, age, cause of death, and risk status. Influenza and Other Respiratory Viruses 2014; 8: 507-515.

25. Zhou H, et al. Hospitalizations associated with influenza and respiratory syncytial virus in the United States, 1993-2008. Clinical Infectious Diseases 2012; 54: $1427-1436$.

26. Thompson WW, et al. Time-series analyses of count data to estimate the burden of seasonal infectious diseases. Epidemiology 2012; 23: 839-842.

27. Yang L, et al. Validation of statistical models for estimating hospitalization associated with influenza and other respiratory viruses. PLOS ONE 2011; 6: e17882.

28. Gay NJ, et al. Estimating deaths due to influenza and respiratory syncytial virus. Journal of the American Medical Association 2003; 289: 2499.

29. Goldstein E, et al. Improving the estimation of influenza-related mortality over a seasonal baseline. Epidemiology 2012; 23: 829-838.

30. Christenson B, et al. Additive preventive effect of influenza and pneumococcal vaccines in elderly persons. European Respiratory Journal 2004; 23: 363-368.

31. Cocoros NM, et al. Obesity as a risk factor for severe influenza-like illness. Influenza and Other Respiratory Viruses 2014; 8: 25-32.

32. Mertz D, et al. Populations at risk for severe or complicated influenza illness: systematic review and metaanalysis. British Medical Journal 2013; 347: f5061.

33. Heikkinen T, et al. Burden of influenza in children in the community. Journal of Infectious Disease 2004; 190: 1369-1373.

34. Mulpuru S, et al. Influenza infection screening tools fail to accurately predict influenza status for patients during pandemic H1N1 influenza season. Canadian Respiratory Journal 2013; 20: e55-59.

35. Michiels B, et al. Clinical prediction rules combining signs, symptoms and epidemiological context to distinguish influenza from influenza-like illnesses in primary care: a cross sectional study. BMC Family Practice 2011; 12: 4.

36. Hawker JI, et al. Trends in antibiotic prescribing in primary care for clinical syndromes subject to national recommendations to reduce antibiotic resistance, UK 1995-2011: analysis of a large database of primary care consultations. Journal of Antimicrobial Chemotherapy 2014; 69: 3423-3430.

37. Heikkinen T, et al. Effectiveness of intranasal live attenuated influenza vaccine against all-cause acute otitis media in children. Pediatric Infectious Disease Journal. 2013; 32: 669-674. 